\title{
Interference between electric and magnetic concepts in introductory physics
}

\author{
Thomas M. Scaife* and Andrew F. Heckler ${ }^{\dagger}$ \\ Physics Department, The Ohio State University, 191 West Woodruff Avenue, Columbus, Ohio 43210-1117, USA
}

(Received 5 October 2010; published 28 March 2011)

\begin{abstract}
We investigate student confusion of concepts of electric and magnetic force. At various times during a traditional university-level course, we administered a series of simple questions about the direction of force on a charged particle moving through either an electric or a magnetic field. We find that after electric force instruction but before magnetic force instruction most students answer electric force questions correctly, and we replicate well-known results that many students incorrectly answer that magnetic forces are in the same direction as the magnetic field. After magnetic force instruction, most students answer magnetic force questions correctly, but surprisingly many students incorrectly answer that electric forces are perpendicular to electric fields, as would happen if a student confused electric forces with magnetic forces. As a further indication of interference between electric and magnetic concepts, we also find that students' responses depend on whether electric or magnetic force questions are posed first, and this effect depends on whether electric or magnetic force was most recently taught.
\end{abstract}

DOI: 10.1103/PhysRevSTPER.7.010104

PACS numbers: 01.40.Fk, 01.40.Ha, 01.50.Kw

\section{INTRODUCTION}

Electricity and magnetism are very similar and closely related phenomena. To an expert, their similarity is illustrated, for example, by their somewhat symmetric presence in Maxwell's equations and the Lorentz force equation. To a novice, electricity and magnetism may also be seen as very similar, but for different reasons. Electricity and magnetism have many analogous surface features, such as polarity (positive-negative and north-south) and the occurrence of both attractive and repulsive forces (magnets sticking to a refrigerator or static repulsion of hair). There is evidence suggesting that these simple similarities may be driving student difficulties in distinguishing between the phenomena of electricity and magnetism. For example, many students (incorrectly) believe that magnetic poles carry electrostatic charges $[1,2]$ or that magnetic fields originate from stationary charged particles [2]. Some consequences of this confusion are that students easily confuse electric and magnetic fields [2,3] and are inclined to (incorrectly) answer that the force experienced by a moving charged particle is directed toward a magnetic pole [4-7] or is in the direction of the magnetic field lines [5-11].

The studies discussed above highlight the fact that many students use the concepts and representations learned during instruction in electricity (which typically occurs prior to magnetism) to answer questions about magnetism. However, is the converse also true? Once students learn

\footnotetext{
*scaife.7@osu.edu

†heckler@mps.ohio-state.edu
}

Published by American Physical Society under the terms of the Creative Commons Attribution 3.0 License. Further distribution of this work must maintain attribution to the author(s) and the published article's title, journal citation, and DOI. about magnetism, will they apply magnetic concepts and representations to answer questions involving electricity? If student difficulties stem from a lack of distinction between electric and magnetic concepts, one might expect a bidirectional confusion (i.e., there are situations in which students apply concepts associated with electricity to answer questions of magnetism, but also situations in which students use concepts associated with magnetism to answer questions of electricity). In this study, we investigated this possibility. In order to allow for systematic comparisons of interference between the two topics, we study the extent to which learning about electric force interfered with responses to questions about magnetic force (replicating earlier findings) as well as the reverse effect in which learning about magnetic force interfered with responses to questions about electric force. We begin with a description of the design of the study.

\section{EXPERIMENTAL DESIGN}

Multiple choice questions were posed to students in either paper and pencil or computer-based format and were varied in four ways. First, students were asked both electric and magnetic force questions. Second, these questions were administered to different populations of students at three times: preelectric and premagnetic instruction, postelectric but premagnetic instruction, and postelectric and postmagnetic instruction. Third, because we previously found that students' responses to magnetic force questions depend upon the representation of the field [7], questions were posed in one of two representations: the field line representation, in which electric and magnetic fields were represented as field lines, a series of arrows that point in the direction of the field, or the pole representation, in which charged plates represented an electric field and magnetic 
poles represented a magnetic field. Fourth, in order to understand better the extent to which the concepts of electric and magnetic force may interact or interfere with each other, the order of questions was varied so that students were either presented with electric force questions before magnetic or magnetic force questions before electric.

\section{A. Participants}

Participants in this study were enrolled in either the mechanics or the electricity and magnetism (E\&M) portion of the calculus-based, introductory physics sequence at The Ohio State University. Data were taken from students who participated as a required part of their course grade. The data were collected over a nonconsecutive sequence of four quarters in order to reduce the possibility of retesting students who enrolled in E\&M immediately after completing mechanics. Upon participation, students were given full credit for either one laboratory quiz or one homework assignment depending on the quarter during which they participated. Although they were not graded on performance, observations of students during the task and exit interviews indicate that the students took the tasks seriously and considered questions carefully before answering.

\section{B. Materials}

After receiving basic training in the standard notation for three-dimensional vectors, participants were asked to determine the direction of force experienced by a positively charged particle moving through either an electric or magnetic field [12]. The field was represented with either poles (i.e., charged plates represent the presence of an electric field and magnetic poles represent the presence of a magnetic field) or field lines.

Students who received the field line representation were asked four electric force questions and four magnetic force questions. Within each type the field pointed toward the top of the page, to the right of the page, into the page, and out of the page. Examples of the questions and the accompanying illustrations are shown in Fig. 1.

Students who received the pole representation were asked two electric force questions and two magnetic force questions. Within each type, the field pointed toward the top and to the right of the page. Since the depiction of a field that points into or out of the page using a pole representation is not easily intelligible, these directions were excluded. Examples of the questions and the accompanying illustrations are shown in Fig. 2.

To control for potential effects due to either the direction or the type of field, students were randomly assigned to receive the questions in one of four possible orders. The orders were counterbalanced so that electric force questions and magnetic force questions were each asked first in two of the four orders.

Because of experimental limitations, question format differed in some quarters. Most notably, the questions described in Secs. III and IV were administered as pencil and paper tasks. The questions described in Sec. V, however, were administered as computer-based tasks. Students received question prompts on a computer display and

For the following questions, the following arrows are used to indicate

direction: left $\leftarrow$, up $\uparrow$, right $\rightarrow$, down $\downarrow$, into page $x$, out of page $\bullet$.

ELECTRIC FORCE QUESTION

In the following questions, a charged particle is moving through an ELECTRIC field. Choose the direction that best describes the FORCE EXERTED ON THE PARTICLE. You can ignore any effects of gravity. Circle the letter which corresponds to your choice.

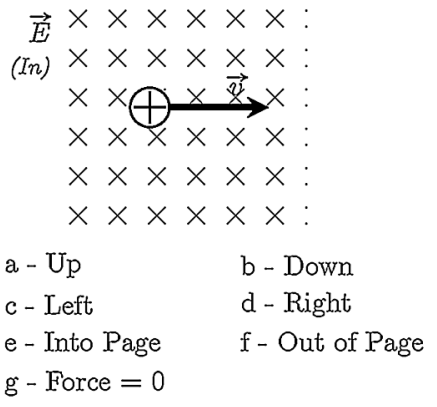

MAGNETIC FORCE QUESTION

In the following questions, a charged particle is moving through a MAGNETIC field. Choose the direction that best describes the FORCE EXERTED ON THE PARTICLE. You can ignore any effects of gravity. Circle the letter which corresponds to your choice.

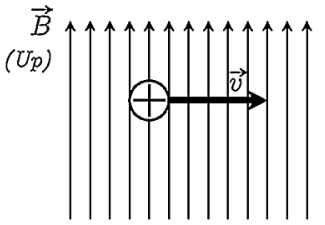

$$
\begin{array}{ll}
a-\text { Up } & b-\text { Down } \\
c-\text { Left } & d-\text { Right } \\
\text { e- Into Page } & \text { f- Out of Page } \\
g-\text { Force }=0 &
\end{array}
$$

FIG. 1. Example showing question text and illustrations received by students who were questioned with the field line representation. Each student answered eight questions in which the direction (up, right, into, and out of) and type (electric and magnetic) of field were varied. 
For the following questions, the following arrows are used to indicate direction: left $\leftarrow$, up $\uparrow$, right $\rightarrow$, down $\downarrow$, into page $\times$, out of page $\bullet$.

Electric Force Question

In the following questions, a charged particle is moving between CHARGED PLATES.

Choose the direction that best describes the FORCE EXERTED ON THE PARTICLE.

You can ignore any effects of gravity. Circle the letter which corresponds to your choice.

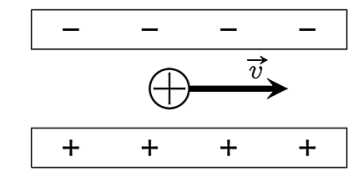

$\begin{array}{ll}\text { a - Up } & \text { b - Down } \\ \text { c - Left } & \text { d - Right } \\ \text { e - Into Page } & \text { f - Out of Page } \\ \mathrm{g}-\text { Force }=0 & \end{array}$

Magnetic Force Question

In the following questions, a charged particle is moving between MAGNETIC POLES. Choose the direction that best describes the FORCE EXERTED ON THE PARTICLE. You can ignore any effects of gravity. Circle the letter which corresponds to your choice.

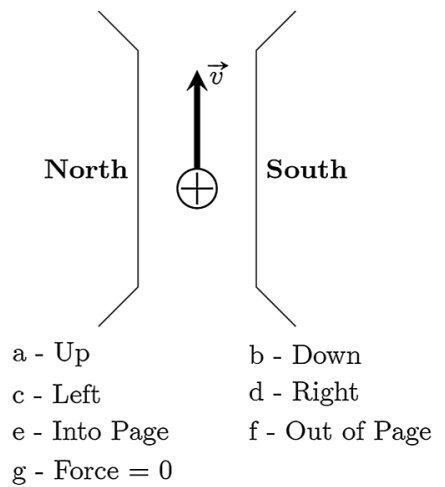

FIG. 2. Example showing question text and illustrations received by students who were questioned with the pole representation. Each student answered four questions in which the direction (up and right) and type (electric and magnetic) of field were varied.

responded by pressing a key on a standard computer keyboard.

\section{Data}

Students' answers were classified into five different categories, in which both positive and negative directions were included in the same categories. For example, the category $\vec{F}$ in direction of $\vec{E}$ includes answers that were both parallel and antiparallel to the electric field. Generally, of those answering in the direction of the field, $10 \%-20 \%$ answered in the direction antiparallel to the field, of those answering in the direction of motion, $0 \%-10 \%$ answered in the direction antiparallel to the velocity of the particle, and of those answering in the direction perpendicular to the field, about $20 \%-30 \%$ answered in the direction antiparallel to the direction of the cross product velocity cross field [13].

\section{PREELECTRIC, PREMAGNETIC INSTRUCTION}

We first administered electric and magnetic force questions to students enrolled in mechanics, the class which precedes $\mathrm{E} \& \mathrm{M}$, in order to determine if confusion between electric and magnetic concepts exists prior to instruction. Although we did not determine how many of these students received instruction in $\mathrm{E} \& \mathrm{M}$ in a high school physics course, none had received E\&M at the university level prior to testing. Students were randomly assigned to one of four conditions in which they were asked either electric or magnetic force questions using either the field line or the pole representation. This between-student design was chosen in order to eliminate any possible effects of interference between electric and magnetic force questions and representation of field. Student responses to the field line and pole representations are shown in Fig. 3.

Inspection of these graphs reveals that many students answer that the force on a charged particle is in the same direction as the field. This result is true for both electric and magnetic fields in both field line and pole representations and it replicates previous research which showed that students answer magnetic force questions similar to electric force questions (see, for example, Ref. [7]). Although this observation is consistent with some students not distinguishing between electric and magnetic forces, we cannot determine whether the answers originate from general confusion or from some other cause, such as the idea that magnets attract nearby objects [1].

While there is no difference in answering patterns between the field line or pole representations for the electric force questions, there is for the magnetic force questions. When questioned using magnetic field lines, most students answer with a force in the direction of field, but when questioned using magnetic poles, the majority of the students answer that the force is in the direction of the motion of the particle. One possible explanation for this answer is that many students may focus only on surface features and choose the direction that is most explicitly depicted in the diagram. Specifically, the field line representation presents a whole series of arrows pointing in the direction of the 


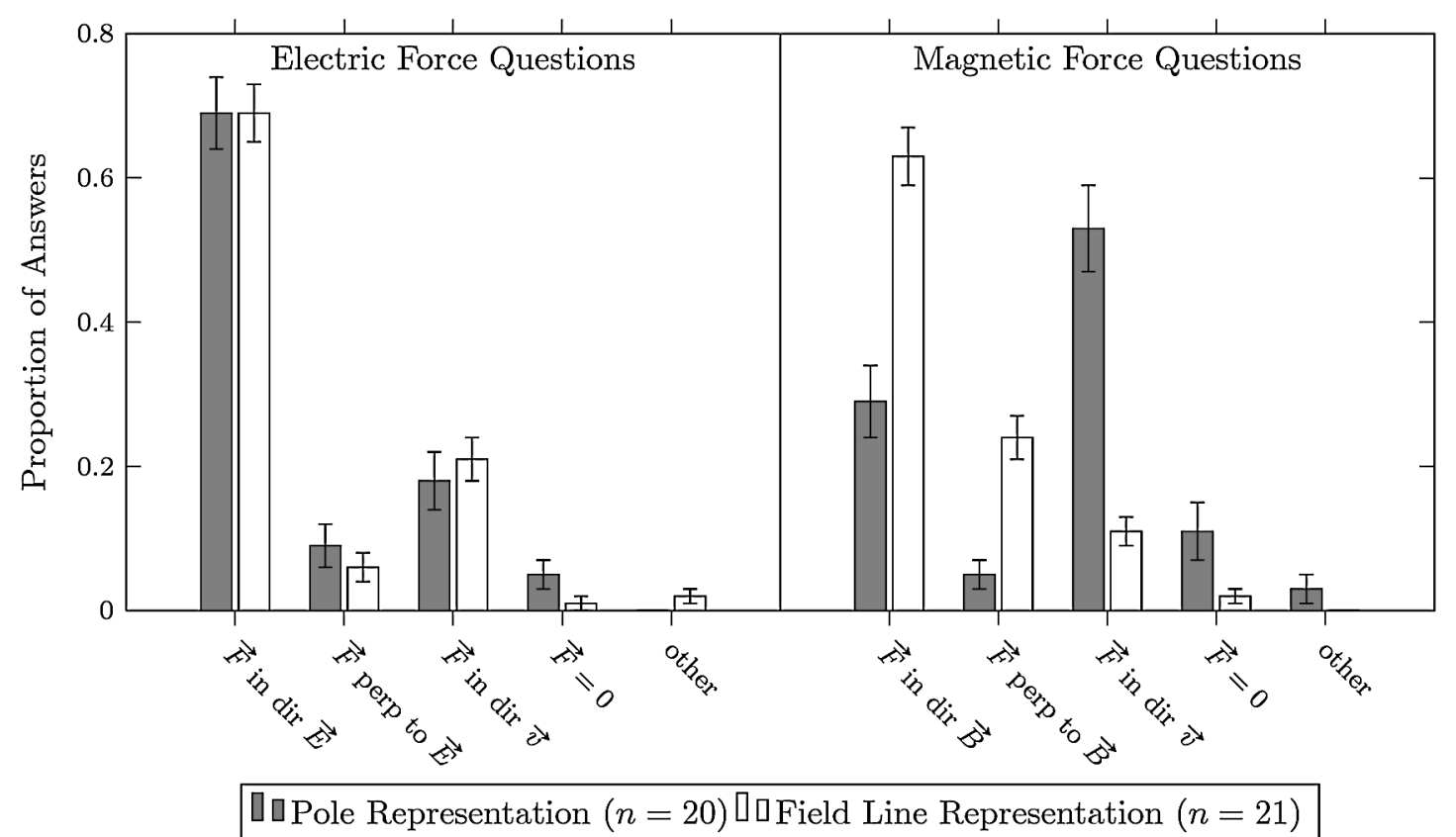

FIG. 3. Student responses to electric and magnetic force questions previous to instruction in both electricity and magnetism.

field, while the pole representation explicitly depicts only one direction with an arrow, the direction of motion. Thus prior to instruction, many students may be uncertain about magnetic forces and simply choose the direction in which the arrows in the diagram are pointing.

In addition to the incorrect answers, when the magnetic field is represented with field lines, a surprisingly large proportion of students (about 25\%) answer correctly that the force is perpendicular to the magnetic field. This representation seems to remind some students about the cross product, the right-hand rule, or some other idea learned previously (perhaps in high school physics) that the magnetic forces are perpendicular to the field. This reminder appears to be lacking from the pole representation, which is consistent with the results of [7].

\section{POSTELECTRIC, POSTMAGNETIC INSTRUCTION}

In order to determine whether instruction about magnetic force interferes with how students answer electric force questions, a simple test was administered to students

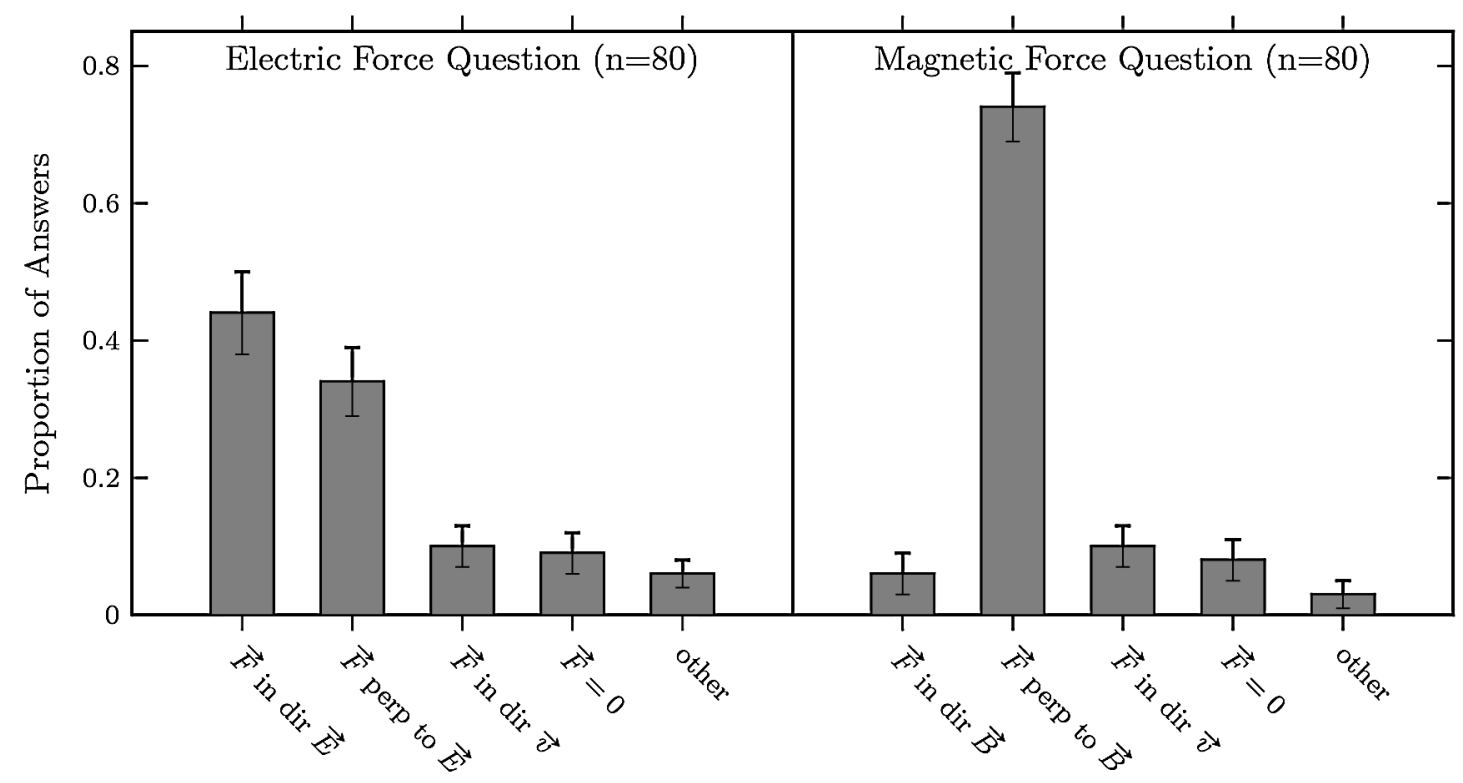

FIG. 4. Student responses to an electric and magnetic question following instruction in both electricity and magnetism. The fields were represented with electric and magnetic field lines, respectively. 
after they had received instruction in magnetism. Students were asked to indicate the direction of the force experienced by a charged particle that was moving through either an electric or a magnetic field that was represented with field lines and directed into the page, similar to the question in Fig. 1. Data relating to the pole representation were not collected as a part of this experiment. The results are presented in Fig. 4. Students answered correctly that the magnetic force is perpendicular to the field, but surprisingly, many students also answered that the electric force is perpendicular to the field.

Comparing these results to the pre-E\&M data that were presented in Sec. III, it appears that instruction was very successful in teaching students to determine the correct direction of magnetic force acting on a charged particle. However, this instruction has also led students to apply the same rules when they answer questions about electric force. Therefore, the overall pattern is that previous to any instruction some students answer magnetic force questions as though they were electric, and following instruction in both electricity and magnetism, some students answer electric force questions as though they were magnetic. These data suggest that the confusion between electricity and magnetism is not limited to answering magnetic force questions as though they were electric.

However, the results from this section and the previous are incomplete. We have not yet described how students answer electric and magnetic force questions after instruction about electricity but before instruction about magnetism. Therefore we are unable to make any conclusions about the effects of electric force instruction alone. The next section will address this issue.

\section{EFFECTS OF INSTRUCTION: POSTELECTRIC, PREMAGNETIC VERSUS POSTELECTRIC, POSTMAGNETIC}

With the data collected from this final experiment, we would like to replicate and extend the findings of the previous sections. Specifically, while the postelectric, postmagnetic data described in Sec. IV showed that magnetic instruction can interfere with the answering of electric force questions, the effect of electric instruction alone has not been determined. Therefore, in this section we report the results of electric and magnetic force questions posed in either the field line or pole representation after electric instruction but before magnetic instruction to one group of students and after magnetic instruction to a separate group of students. Although postmagnetic data were already reported in Sec. IV, the measurements were repeated here to allow direct comparisons from before to after magnetic instruction.

\section{A. Field line representation}

As described in Sec. II, students were presented with diagrams depicting a charged particle moving through an electric or magnetic field and asked to choose the direction that describes the force on the particle. Student responses to questions that used the field line representation are shown in Fig. 5. These results have been averaged across

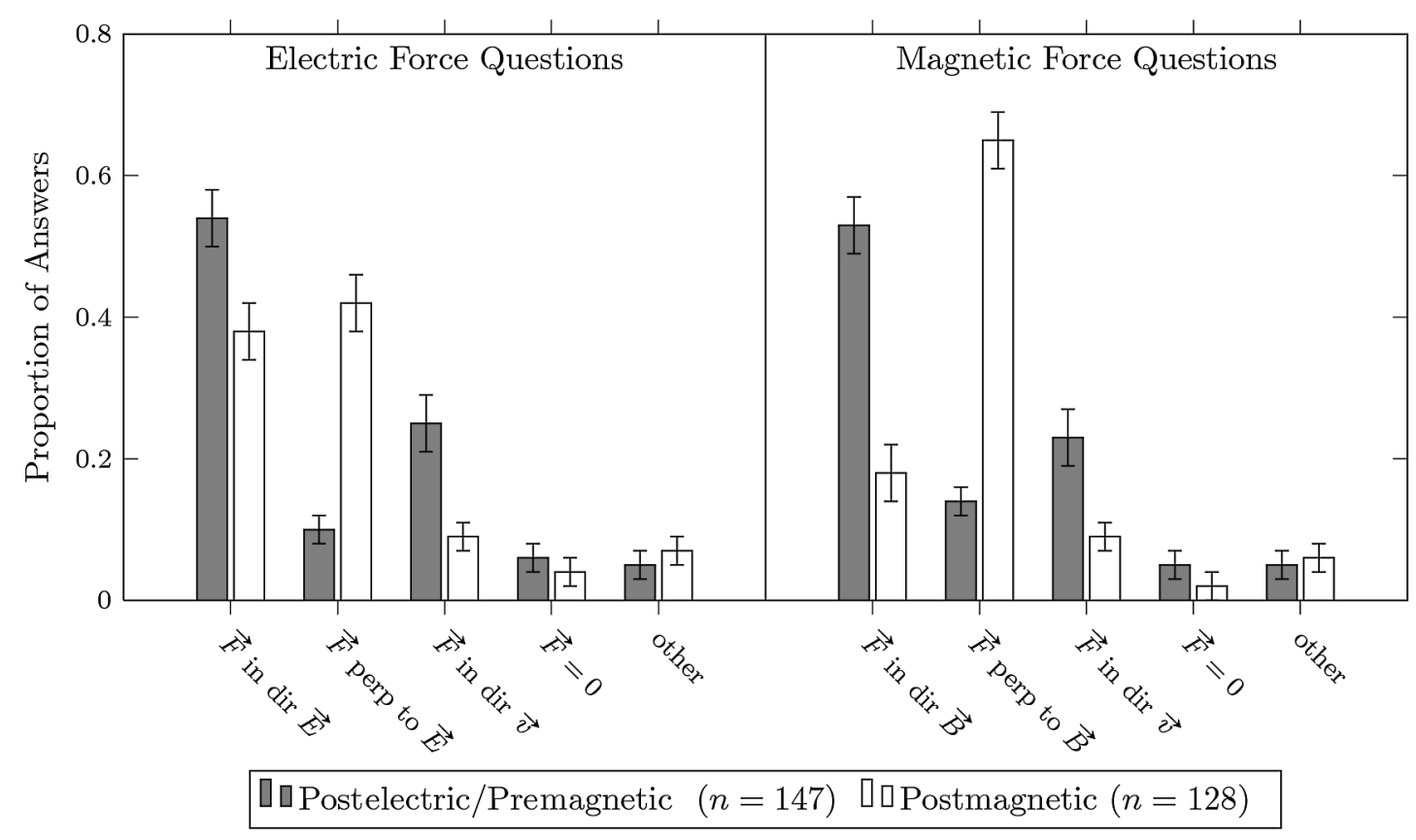

FIG. 5. Results showing how students' answers to electric and magnetic force questions changed from before to after instruction in magnetism. Fields were depicted using field lines. 
all question orders, including orders in which the electric field questions were posed first and orders in which the magnetic field questions were posed first. Any specific effects of question order will be addressed in Sec. V C.

The results show that immediately following instruction in electricity, $\sim 55 \%$ of students answer the electric force question correctly and $\sim 50 \%$ incorrectly answer the magnetic force questions with a force in the direction of the field - as though they were electric force questions. Immediately following instruction in magnetism, $\sim 70 \%$ of students answer the magnetic force questions correctly and $\sim 45 \%$ incorrectly answer that the electric force is perpendicular to the electric field - as if particles in electric fields obeyed the recently learned magnetic Lorentz force rule. These changes in student responses from before to after magnetic force instruction are statistically reliable (electric force questions: $\chi^{2}=168.10, d f=4, p<1 \times 10^{-6}$; magnetic force questions: $\chi^{2}=324.87, d f=4, p<1 \times 10^{-6}$ ) and correspond to the sharp increases in answers perpendicular to the field (electric force questions: effect size [14] $d=0.78$; magnetic force questions: $d=1.24$ ) [16]. Before magnetic force instruction, students answer that electric force is in the direction of electric field lines. After instruction in magnetism, when the presence of a field is represented by field lines, students answer that the force experienced by a charged particle is perpendicular to the field, regardless of the type of field.

There are a couple of issues to note from these results. First, the fraction of correct answers on the electric force questions directly after electric instruction is surprisingly low (55\%). This could be due to students' lack of familiarity with field lines (see also a discussion in the Sec. VI). Regardless, such a low score following instruction may indicate that this representation is vulnerable to interference. Second, since these tests were administered no more than two weeks after magnetic force instruction, the observed interference may only be temporary or it may persist for months or years after instruction. These data cannot predict the longevity of the interference.

\section{B. Pole representation}

Student responses to the pole representation are shown in Fig. 6. Again, these results have been averaged across all question orders, including orders in which the electric field questions were posed first and orders in which the magnetic questions were first.

There are three noteworthy observations to be made from Fig. 6. First, and most importantly, unlike in the field line representation, magnetic force instruction does not significantly interfere with students' answers to the electric force questions. A large fraction of students $(\sim 70 \%)$ answer the electric force question correctly, both before and after magnetic force instruction. Only a small fraction of students $(\sim 10 \%)$ answered incorrectly that the electric force was perpendicular to the field after instruction in magnetism. Comparing the postmagnetic instruction proportions of correct electric force answers in Figs. 5 and 6, it appears that a larger fraction of students answered correctly in the pole representation than the field line representation ( $70 \%$ vs 55\%), which may be because students are more familiar with charged plates than field lines. In any case, the larger fraction of correct answers suggests

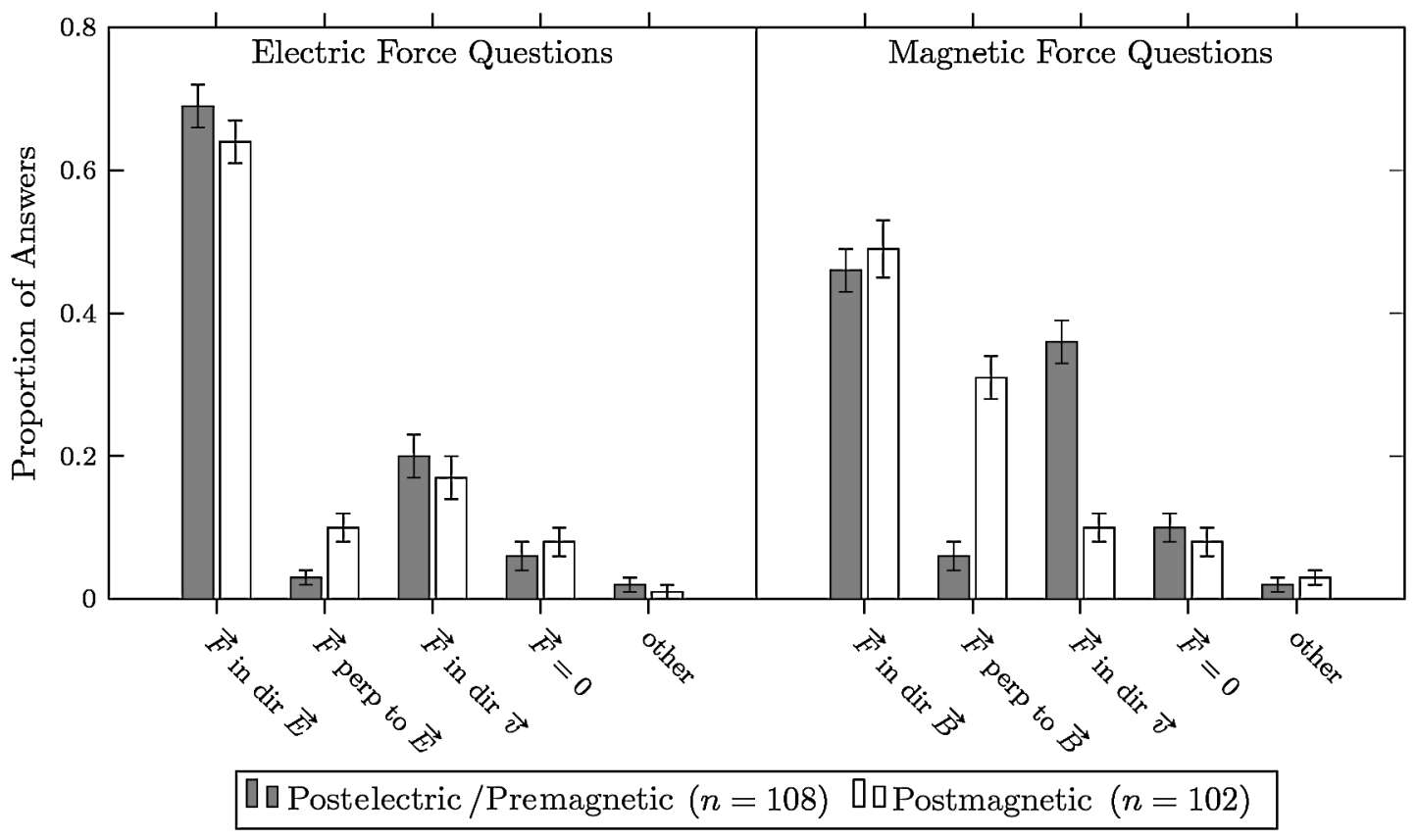

FIG. 6. Results showing how students' answers to electric and magnetic force questions changed from before to after instruction in magnetism. Fields were depicted using poles. 
that it is less vulnerable to interference than the field line representation.

Second, when the presence of an electromagnetic field is represented by a pole, instruction in magnetism does not reduce student answers in the direction of the field. Therefore, the idea that charged particles experience forces that are directed toward or away from a pole appears to be resistant to standard instruction. When the magnetic force answers are examined, the force in the direction of field answers remained the same $(d=0.07)$, whereas the force in the direction of motion answers decreased $(d=-0.66)$. This is contrasted against the field line representation where the force in the direction of field answers decreased $(d=-0.77)$ as did the force in the direction of motion answers $(d=-0.41)$.

Third, there is a significant difference in magnetic force answers in the pole representation from premagnetic to postmagnetic force instruction $\left(\chi^{2}=64.25, d f=4, p<\right.$ $1 \times 10^{-6}$ ) resulting from the increase in correct answers ( $5 \%$ to $35 \%, d=0.67)$.

In addition, when comparing the results in the pole representation to those of the initial postmagnetic experiment (Fig. 4), it is clear that students presented in Fig. 6 did not answer questions with a force perpendicular to the field as frequently as the students presented in Fig. 4. This could be due to a difference in instructors or the student

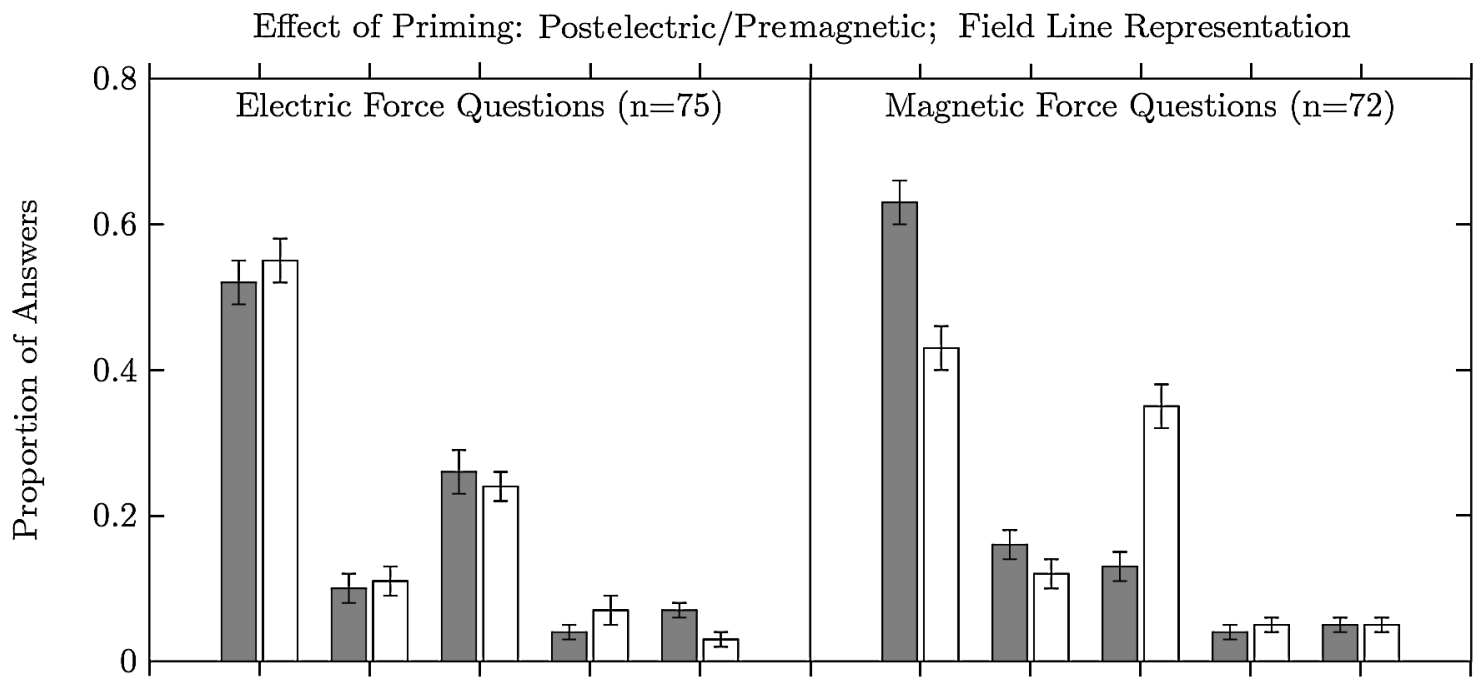

Effect of Priming: Postelectric/Postmagnetic; Field Line Representation

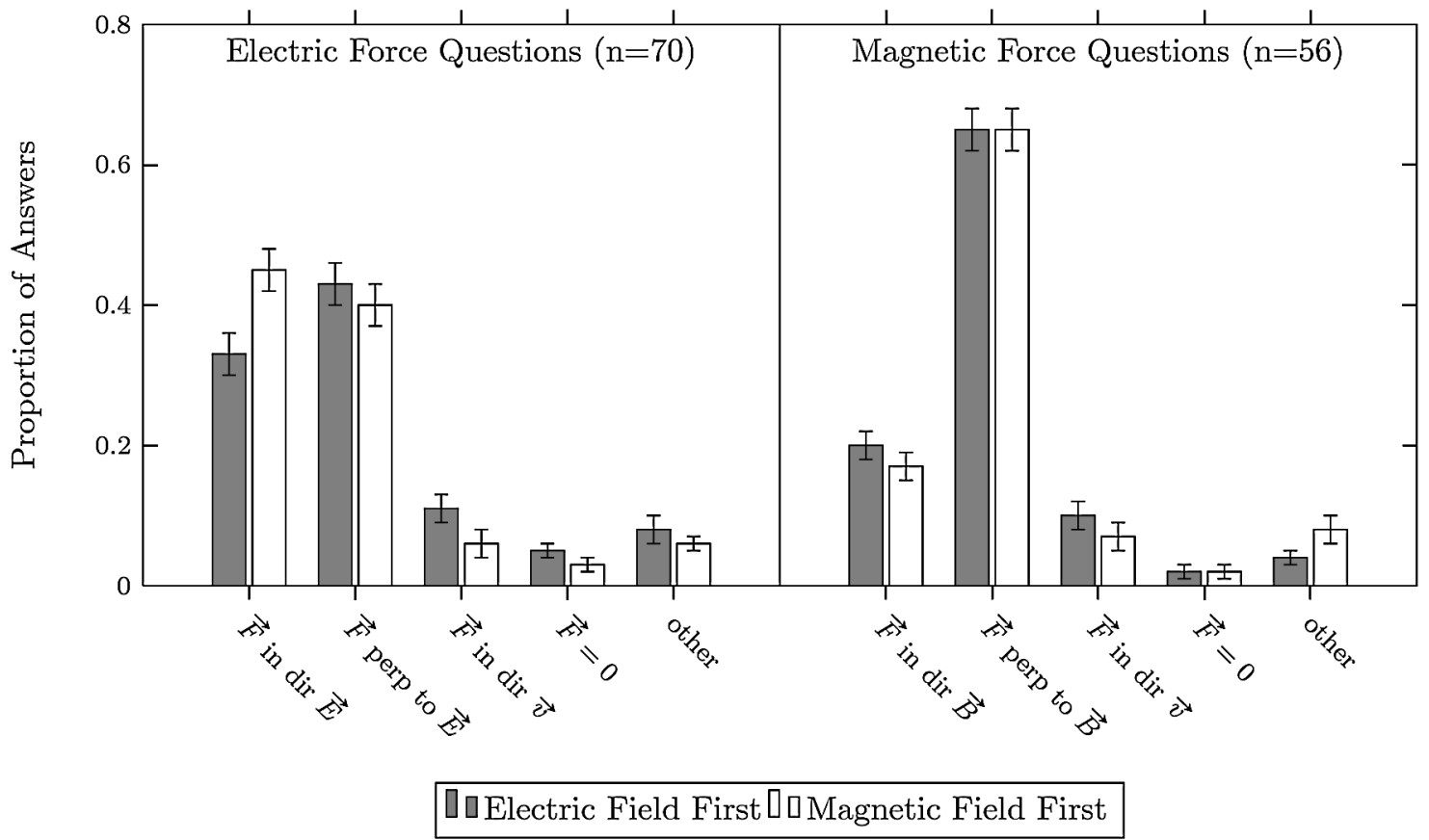

FIG. 7. Student responses showing the effect of question order (or cognitive priming) on the field line representation. 
populations that were tested in each experiment. The vital data that are consistent across the two experiments is that following instruction in magnetism some students do correctly answer that the magnetic force on a moving charged particle is perpendicular to the field.

\section{Priming: Presenting electric or magnetic force questions first}

A second method that can be employed to determine the extent to which students are confusing concepts of electricity and magnetism is to examine the effects of question order on student answering; will there be a difference in student answering if the electric force questions are posed before the magnetic force questions as opposed to after? Differing answer distributions would be an indication of the phenomenon "cognitive priming," in which exposure to one stimulus (in this case electric or magnetic force questions) will influence the response to a subsequent stimulus (for example, see Ref. [17]). Since priming can occur when the first stimulus is perceived as similar to the second, we are likely to observe priming if the students perceive perceptual or conceptual similarities between electricity and magnetism. Therefore, if students did not have difficulties differentiating between electric and magnetic force questions, we would expect question order to have little effect on the answer patterns. If the difficulties

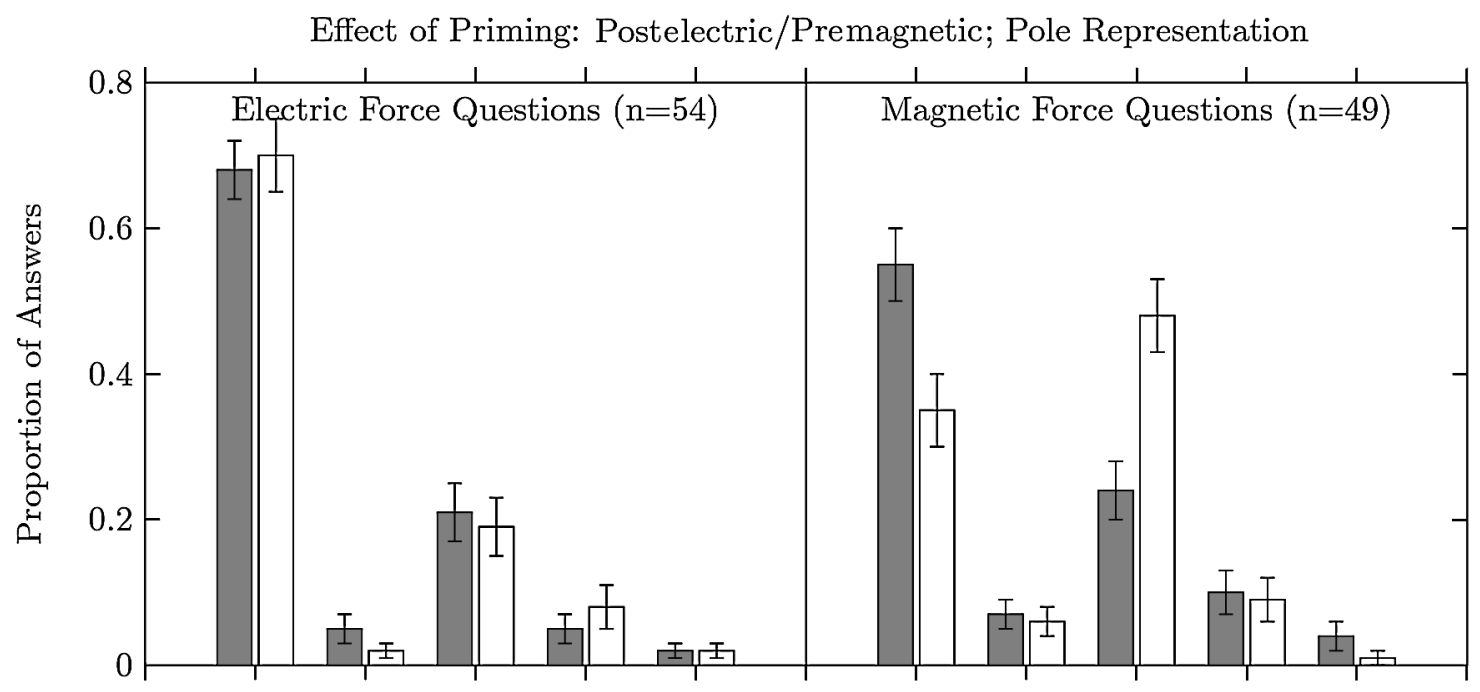

Effect of Priming: Postelectric/Postmagnetic; Pole Representation

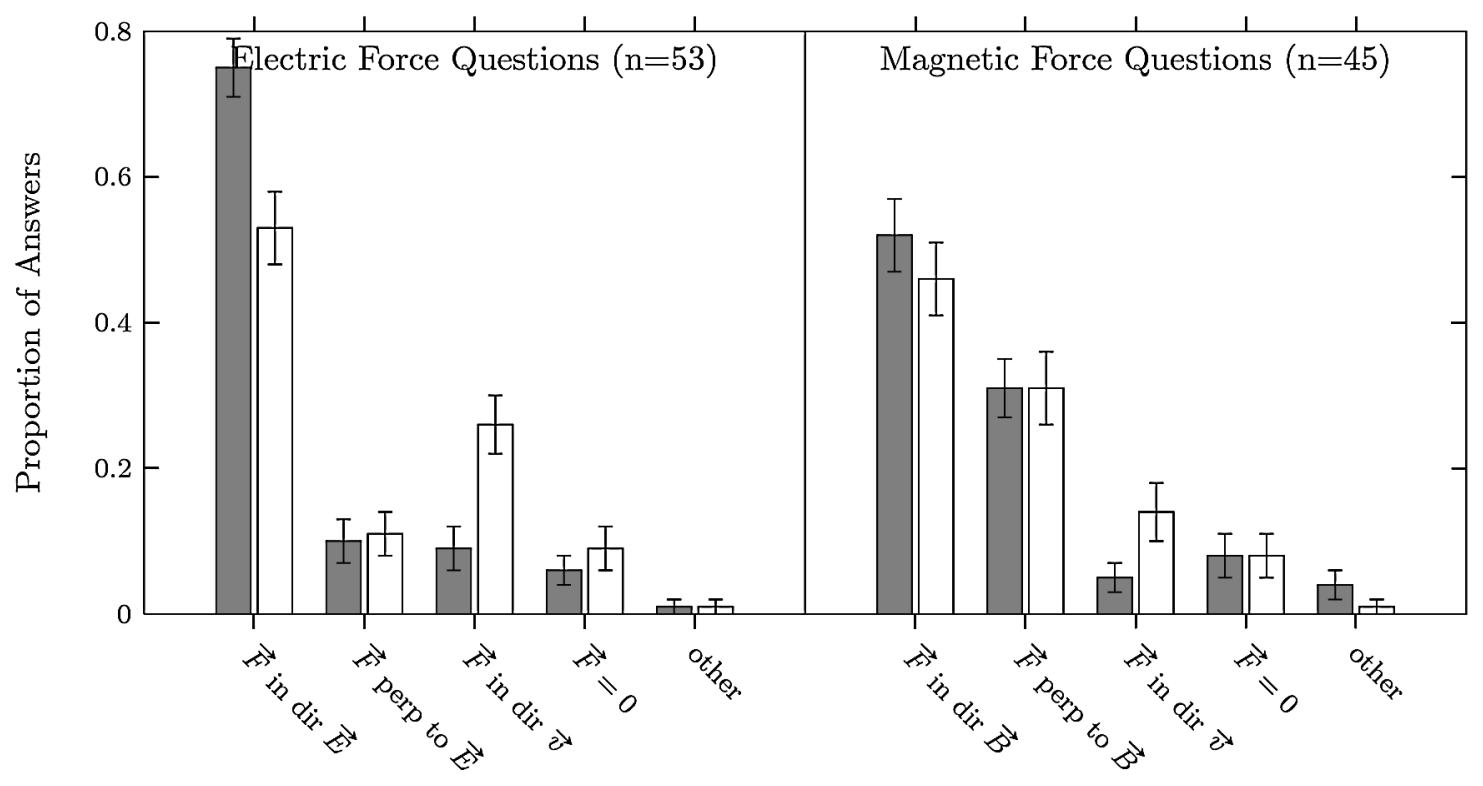

पaElectric Field First QuMagnetic Field First

FIG. 8. Student responses showing the effect of question order (or cognitive priming) on the pole representation. 
were unidirectional, where, for example, students answer magnetic questions as though they were electric, we would expect that question order affects responses to magnetic questions but not electric questions. Finally, if student difficulties were bidirectional, that is electric and magnetic concepts interfere with each other, we would expect that question order would affect responses to both electric and magnetic questions.

As mentioned earlier, Figs. 5 and 6 present data averaged over question orders. Figures 7 and 8 redisplay the field line representation and pole representation response patterns, respectively. In the latter figures, the data have been divided into two order conditions: one in which electric force questions were posed before magnetic force questions, and the other in which magnetic force questions were posed before electric force questions. While Figs. 5 and 6 include all student responses (e.g., some students might have only answered the electric force questions, ignoring the magnetic force questions), Figs. 7 and 8 only include responses if students answered all questions. In order to compare different question orders, it is necessary to only examine students who completed the entire question sequence.

The most important result from Figs. 7 and 8 is that both representations share a similar effect: changing question order does not affect student answering for the topic most recently taught, but it does affect answering for the "other" topic. For example, immediately following electric force instruction, question order does not affect how students answer electric force questions in either the field line $\left(\chi^{2}=7.65, d f=4, p=0.11\right)$ or the pole $\left(\chi^{2}=\right.$ $2.15, d f=4, p=0.71)$ representations. However, question order does affect how students answer magnetic force questions in the field line $\left(\chi^{2}=43.29, d f=4, p<\right.$ $\left.1 \times 10^{-6}\right)$ and the pole $\left(\chi^{2}=14.84, d f=4, p<0.01\right)$ representations. In both cases, asking the electric force questions first led more students to answer magnetic force questions as if they were electric force questions.

Analogously, immediately following magnetic force instruction, question order did not affect responses to magnetic force questions for the field line $\left(\chi^{2}=6.25, d f=4\right.$, $p=0.18)$ or the pole $\left(\chi^{2}=5.88, d f=4, p=0.21\right)$ representations, but it did affect answering for electric force questions in the field line $\left(\chi^{2}=12.2, d f=4\right.$, $p=0.02)$ and the pole $\left(\chi^{2}=13.77, d f=4, p<0.01\right)$ representations.

The data suggest that interference between electric and magnetic force questions is at any given time unidirectional, with the direction depending on which force was most recently taught. Because priming was observed for both representations and for both electric and magnetic force questions, students likely perceive the questions to be similar at the perceptual level (e.g., charged plates and magnetic poles or electric field lines and magnetic field lines are perceptually similar), the conceptual level (electric and magnetic force are conceptually similar), or both. Furthermore, because the effects of question order changes with respect to instruction, the perceived similarity between electric and magnetic forces is also affected by instruction.

The way in which question order affected magnetic force questions prior to magnetic force instruction is straightforward: electric force questions tended to cue students to choose electric-force-like responses. However, it is not clear why, after magnetic force instruction, answering magnetic force questions first tended to cue students to answer electric force questions more correctly in the field line representation and more incorrectly in the pole representation.

\section{CONCLUSIONS AND IMPLICATIONS FOR INSTRUCTION}

The most important and surprising finding of this study is that, directly after instruction about magnetism, many students answer that the direction of the force on a charged particle moving through an electric field is perpendicular to the electric field, presumably by employing the same right-hand rule that was learned for magnetic forces. Thus, despite the fact that directly before magnetic force instruction students were answering electric force questions correctly, up to two weeks (and possible longer) after they learn about magnetic force, they answer electric force questions as though they were magnetic force questions. We have framed this result as analogous to the wellestablished converse observation that after instruction about magnetism many students answer that the magnetic force on a charged particle is either in the direction of magnetic field or directed towards a magnetic pole, similar to the rule for electric force. Therefore, we have observed a symmetric behavior in interference between concepts of electric and magnetic fields and forces, and the interference was strongly influenced by which topic was most recently taught. The data we have presented do not describe the extent to which, months or years after instruction, students will continue to choose the magnetic-forcelike response for the electric force question. As memory of the magnetic force rule presumably fades with time, it is likely that the electric force responses would revert back to premagnetic instruction patterns.

Through examination of student response patterns, we have also found that interference between the concepts of electric and magnetic force can depend on whether a field line or pole representation is used. Specifically, after magnetic force instruction, students tend to answer incorrectly that the electric force is perpendicular to the electric field more often in the field line representation than in the pole representation. Conversely, following magnetic force instruction, more students continue to answer incorrectly that the magnetic force is in the direction of the magnetic field when the field is represented with magnetic poles. This 
latter result is consistent with previous work as reported in Ref. [7].

There are a number of possible explanations for the observed answer distributions, all of which are centered around the general idea that, because electricity and magnetism have many similar surface features, students either explicitly or implicitly perceive questions about the two as similar (or even identical). One possibility is that many students explicitly believe that electric and magnetic fields and forces are identical. This possibility does not appear consistently to explain the results of this study since the response patterns change both with instruction and with representation. Another possibility is that many students are implicitly confusing electric and magnetic concepts because they are not aware that there is a need to distinguish between the two concepts. Finally, it is possible that students are aware that there are differences between electric and magnetic forces when prompted, but they rarely recognize that this knowledge is needed when answering electric or magnetic force questions. Instead, they confuse electric and magnetic forces because they respond based on the surface features of the problem and what is most available in memory (for example, what the students most recently learned).

We propose that the final possibility, that most students understand that there are differences but largely base their answers on surface features of the question, is the most likely (cf. Ref. [18]). This claim is supported by our general observation that in the courses studied, electric force questions were most often represented in the context of charged plates (i.e., poles) and not field lines, and magnetic force questions were most often represented in the context of field lines and not magnetic poles. In fact, a general inspection of the course textbook [19] reveals the same tendency to represent electric fields with charged plates and magnetic fields with field lines. Thus one would expect that students tend to identify electric-force-like answers with pole representations and magnetic-forcelike answers with field line representations, as seen in the answer distributions found in this study.

The results and analyses presented in this study imply several possibilities for instructional change. Consistent with our previous findings [7], the use of field line or pole representation appears to influence significantly what students learn about electric and magnetic fields and forces. The common practice of using charged plates to represent electric fields and field lines to represent magnetic fields during instruction appears to be biasing student responses. One method to combat the resulting lack of distinction between electricity and magnetism might be to make frequent, explicit comparisons between electric and magnetic forces and fields. An important aspect of these comparisons should include translation between both field line and pole representations.

Finally, while it is clear that the standard instruction about magnetic force was fairly effective, as measured by students' responses in the field line representation, it is surprising to see that there is no strong evidence that instruction about electric force improved student understanding in either the field line or pole representations. Before any instruction, $\sim 70 \%$ of students could already correctly answer that the direction of force is either in the direction of the field or towards a charged plate. It is possible that this signal is a false positive since students might intuitively guess that the force is in the direction of the field arrows or in the direction of plates. (The fact that many students also answered this way for magnetic force prior to any instruction further supports the possibility of a false positive.) Yet, directly after electric force instruction, the number of correct responses did not increase for the pole representation and even slightly decreased for the field line representation. Does standard instruction have zero positive effect on student understanding of these questions but a positive effect on magnetic force questions? While it is possible that students did not properly understand the questions or the representations, evidence from exit interviews does not support this claim. Therefore, it remains an open question as to whether instruction had any positive effect on student understanding of the direction of electric force on a particle.

\section{ACKNOWLEDGMENTS}

We would like to thank Rebecca Rosenblatt for assisting with the collection of data and the supervision of participants. In addition, we are grateful for the insightful comments made by the anonymous referees. This work was partially supported by the Institute of Education Sciences under Grant No. 60003571.
[1] A. T. Borges and J. K. Gilbert, Models of magnetism, Int. J. Sci. Educ. 20, 361 (1998).

[2] J. Guisasola, J. M. Almudi, and J. L. Zubimendi, Difficulties in learning the introductory magnetic field theory in the first years of university, Sci. Educ. 88, 443 (2004).
[3] M. Sağlam and R. Millar, Upper high school students' understanding of electromagnetism, Int. J. Sci. Educ. 28, 543 (2006).

[4] D. P. Maloney, Charged poles?, Phys. Educ. 20, 310 (1985). 
[5] D. P. Maloney, T.L. O'Kuma, C. J. Hieggelke, and A. Van Heuvelen, Surveying students conceptual knowledge of electricity and magnetism, Am. J. Phys. 69, S12 (2001).

[6] T. M. Scaife and A.F. Heckler, The effect of field representation on student responses to magnetic force questions, AIP Conf. Proc. 951, 180 (2007).

[7] T. M. Scaife and A.F. Heckler, Student understanding of the direction of the magnetic force on a charged particle, Am. J. Phys. 78, 869 (2010).

[8] G. Aubrecht and C. Raduta, Contrasts in student understanding of simple E\&M questions in two countries, AIP Conf. Proc. 790, 85 (2005).

[9] M. C. Pocovi and F. Finley, Lines of force: Faraday's and students' views, Sci. Educ. 11, 459 (2002).

[10] C. Raduta, General students' misconceptions related to electricity and magnetism, arXiv:physics/0503132.

[11] In addition to these documented difficulties, we have regularly observed students claiming that magnets are attracted to charged objects during classroom interactions.

[12] In order to minimize possible confounding factors, students were not asked to determine the force on negatively charged particles.

[13] For a detailed discussion concerning sign errors made when students are asked to determine the direction of magnetic force, see [7]. Our results are consistent with the findings of this previous study.

[14] The effect size was calculated using Cohen's $d$ with a pooled standard deviation (see, for example, Ref. [15]).

[15] J. Cohen, Statistical Power Analysis for the Behavioral Sciences (Lawrence Erlbaum Assoc., Hillsdale, 1988), pp. 66-67, 2nd ed.

[16] While this effect was first observed as reported by data in this paper, this behavior was also reported and labeled as interference by A. F. Heckler and E. C. Sayre in Ref. [20].

[17] D. Reisberg, Cognition (W.W. Norton \& Company, Inc., New York, 2009), 4th ed.

[18] M. T. H. Chi, P. J. Feltovich, and R. Glaser, Categorization and representation of physics problems by experts and novices, Cogn. Sci. 5, 121 (1981).

[19] D. Halliday, R. Resnick, and J. Walker, Fundamentals of Physics (John Wiley and Sons, New York, 2010), pp. 561-790, 9th ed.

[20] A. F. Heckler and E. C. Sayre, What happens between pre- and post-tests: Multiple measurements of student understanding during an introductory physics course, Am. J. Phys. 78, 768 (2010). 